ERRATA

\title{
TULA: an SH3- and UBA-containing protein that binds to c-Cbl and ubiquitin
}

\author{
Elena A Feshchenko ${ }^{1,4}$, Evgeniya V Smirnova ${ }^{1}$, Gayathri Swaminathan ${ }^{1}$, Anjali M \\ Teckchandani ${ }^{1}$, Rachana Agrawal ${ }^{1}$, Hamid Band ${ }^{2,5}$, Xiaolong Zhang ${ }^{3,6}$, Roland S Annan ${ }^{3}$,
} Steven A Carr ${ }^{3,7}$ and Alexander Y Tsygankov",1

Oncogene (2004) 23, 9449. doi:10.1038/sj.onc.1208320

Correction to: Oncogene (2004) 23, 4690-4706. doi: 10.1038/sj.onc. 1207627

Due to a typesetting error, the author affiliations were published incorrectly. The correct details are as below.

${ }^{1}$ Department of Microbiology and Immunology, Temple University School of Medicine, Philadelphia, PA, USA

${ }^{2}$ Division of Rheumatology, Immunology and Allergy, Brigham and Women's Hospital, Harvard Medical School, Boston, MA, USA
${ }^{3}$ Glaxo-SmithKline, King of Prussia, PA, USA

${ }^{4}$ Current address: Curagen, Branford, CT, USA

${ }^{5}$ Current address: Feinberg School of Medicine, Northwestern University, Evanston, IL, USA

${ }^{6}$ Current address: Celera Genomics, Rockville, MD, USA

${ }^{7}$ Current address: Millennium Pharmaceuticals, Cambridge, MA, USA

*Correspondence: AY Tsygankov, Department of Microbiology and Immunology, Temple University School of Medicine, 3400 N Broad Street, Philadelphia, PA 19140, USA; E-mail: alexander.tsygankov@ temple.edu

\section{SOC1 inhibits HPV-E7-mediated transformation by inducing degradation of $\mathbf{E} 7$ protein}

Masaki Kamio, Takafumi Yoshida, Hisanobu Ogata, Tsutomu Douchi, Yukihiro Nagata, Makoto Inoue, Mamoru Hasegawa, Yoshikazu Yonemitsu and Akihiko Yoshimura

Oncogene (2004) 23, 9449. doi:10.1038/sj.onc.1208310

Correction to: Oncogene (2004) 23, 3107-3115. doi:10.1038/sj.onc. 1207453
Owing to a typesetting error, the title of the above article was published incorrectly. The correct title is 'SOCS1 inhibits HPV-E7-mediated transformation by inducing degradation of E7 protein.' 